EPS is in essence international in character, and its creation and development required a fair amount of international collaboration and goodwill.

\title{
EPS and the International Scene
}

To consider EPS in terms of international relations is to address a tautology. But EPS is in essence international in character and its creation required a fair amount of international collaboration and goodwill. Partnership between eastern and western Europe for a long time looked more difficult than that between the western part of Europe and other parts of the world, in particular North America. Yet there was a deep feeling that the division of Europe into two well-separated pieces would not last for ever and that, despite the difficulties, every effort had to be made to build bridges.

In 1965, when something which grew to become EPS was first talked about, Europe was in a period of relative détente. Conferences and meetings which brought together physicists from the west and from the east were taking place in increasing numbers. I remember, in particular, attending in 1966 a topical meeting beside Lake Balaton in Hungary that was organized by George Marx. The strong collaboration between particle physicists had come of age, and CERN was discussing participation in the experiments on the Soviet Union's new accelerator at Serpukhov while planning its own major extension (a $300 \mathrm{GeV}$ accelerator to be built on a new site). Besides CERN, prestigious centres in Europe such as the Niels Bohr Institute, Copenhagen, and the International Centre for Theoretical Physics, Trieste, were doing a great deal to bring physicists together. A pan-European society thus appeared natural.

\section{Setting in Motion}

The early history of EPS is well known: discussions at an informal dinner with 30 international guests invited by Gilberto Bernardini, the President of the Italian Physical Society, during the annual meeting and Council meeting of the society in Bologna in November 1965 triggered a meeting in Pisa on European Collaboration in Physics in April 1966. Organized by Bernardini under the sponsorship of the President of the Republic of Italy, it was there, in a session chaired by $C$. Møller, that the key question "Should we have a European Physical Society?" was first put in a formal way.

Louis Cohen, the Secretary (chief executive officer) of The Institute of Physics and The Physical Society (IPPS; now simply The Institute of Physics) until his retirement recalls that:

... of the other topics, that of physics journals could be subsumed with the EPS: the future of Il Nuovo Cimento was really a domestic issue of the Italian Physical Society, and scientific policy was more appropriately dealt with by a quasi-political body (e.g., the European Science Foundation established some years later).

There were two external organizations which had an influence on the thinking behind the EPS. These were the International Union of Pure and Applied Physics (IUPAP) and The American Physical Society/American Institute of Physics (APS/AIP). It was accepted that IUPAP had its strengths in being able to organize meetings and programmes on a world-wide

\section{Jacob \\ CERN, Geneva}

basis. It also had its weakness by being unable to effectively involve individual physicists on a regional basis. Consequently, while receiving the moral support of IUPAP, there would be no conflict between the EPS and IUPAP.

The first EPS Vice-President, Erik Rudberg who had been with the National Institute for Metals Research in Stockholm before becoming the Secretary of the Swedish Academy of Sciences, remarked in an unpublished interview with Edwin Shaw in 1968 that:

"There was of course IUPAP but its ambitions were limited and there was no pressure to extend as the International Union of Pure and Applied Chemistry (IUPAC) as the astronomers had done. What impressed me most was the real possibility of making proper contact with physicists in eastern Europe.

Despite accusations ... our best hope for improving the lot of our fellow humans lies through science and the greater understanding it gives to natural phenomena. That is the direct reason for supporting initiatives like EPS.

But we have to move in steps. Too big a jump and you fail. Physics is at the basis of science and we see today a spreading into chemistry, etc. as these fields seek a better theoretical formulation of their concepts. Physics is therefore a natural leader. So you may ask why a European society rather than say an extension of IUPAP. The idea is sound because this region is a "hot spot", a point of confrontation, a point where cultures meet and where issues are visible. We have the success of CERN to enourage us."

Commenting on what he felt were the motivations behind the interest in an EPS, Cohen had gone on to say:

"Looking to the situation in the United States showed what could be done in the organization and coordination of meetings and, above all, in the publication of a range of physics journals which were highly successful, both scientifically and financially. Although the USA was viewed as an effective model, it was realised by some if not all, physicists in Europe that the APS and the AIP were mature organizations. However, it was an undoubted fact that many European physicists were members of the APS in order to gain access, as both authors and readers, to the Phy-

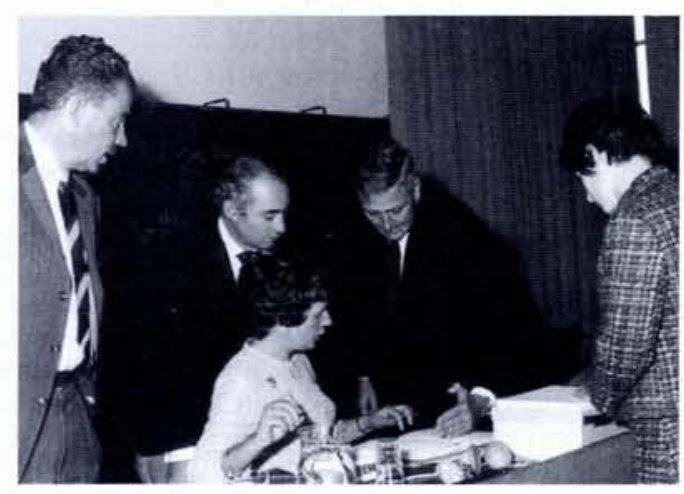

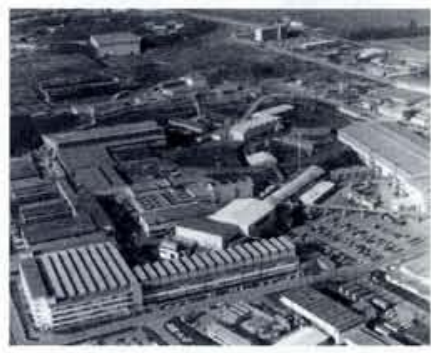

CERN in 1971: in the background is the tidied-up site of the Intersecting Storage Ring which had begun to be constructed in 1967.

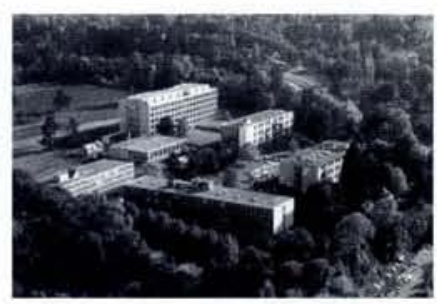

Institut Battelle, Geneva, in the early 1970's when its staff peaked at around 800 . The Battelle Me. morial Institute based in Columbus, Ohio, established laboratories in Geneva and Frankfurt in the 1950 's which saw major expansions in the 1960's (the Institute had a total staff worldwide of 5500 in 1965). The Geneva site remains essentially the same today, while its Battelle staff now numbers some 130 .
Standing from the left, Laurens Jansen, Louis Cohen, and Bernard Gregory at the Plenary Meeting of EPS at CERN in September 1968. Seated is L. Etienne. 


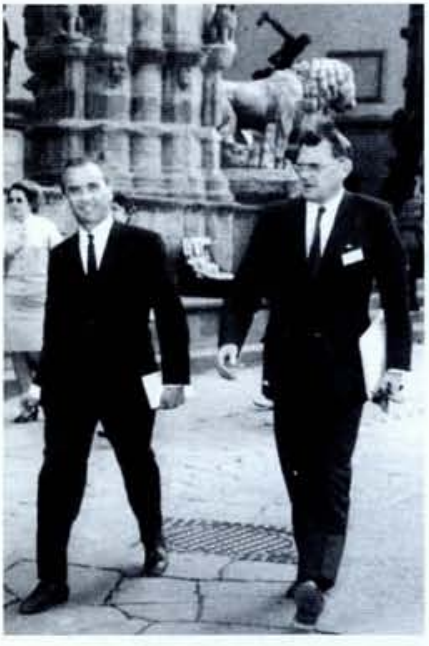

Edgar Ascher (on the left) from the Institut Battelle, who coordinated incoming information for Europhysics News in 1969, and Hugo Thiemann, the Institute's Director, at the Inaugural Conference of the EPS in Florence in April 1969.

Maurice Jacob (EPS President, 1991-93) placing a wreath to commemorate the Centenary of the Eötvös Lorand Physical Society in Budapest in October 1991.

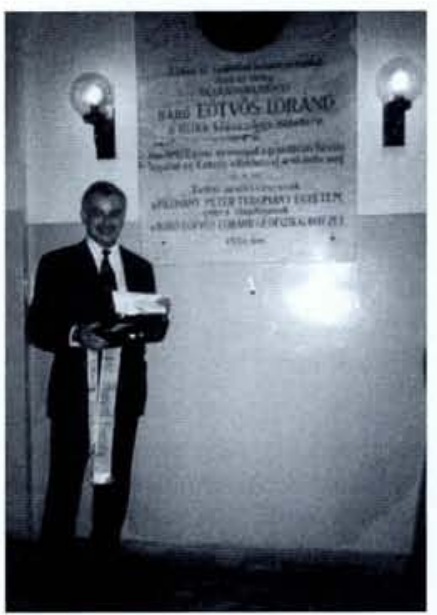

sical Review journals published by APS. Would it not be more satisfactory for a parallel situation to exist in Europe? Nobody was suggesting that there was such a thing as "European physics", but there were physicists living and working in Europe who could be better served by a European organization."

\section{Working Group Formed}

The answer to the deliberations was, as expected, a resolution that "steps should be taken" and acceptance of a resolution calling for a "Working Group for Further Action". Bernardini sent out invitations to join a Steering Committee which first met in November at CERN under the chairmanship of Bernard Gregory, CERN's Director-General. CERN was to join as one of the first Associate Members with a contribution of SFR $20000 .-$, an important sum at the time. Gregory also remained a staunch supporter, remarking in 1970: "You may feel quite sure that whatever help CERN has given to EPS sprang from the strong belief that its rôle would be vital for the development of research in Europe."

Laurens Jansen, the first EPS Secretary, recalls that:

"Somewhere between the first and second meetings, we, as members of the Advanced Studies Centre at Battelle-Geneva joined forces with Bernardini, CERN and the University of Geneva. The feeling that we, and Battelle, should do so was unanimous and very spontaneous, a response to a long awaited and absolutely essential development. Reminiscing, my personal motivation probably stemmed from a (positive) six-years' experience in the United States. I returned to Europe a European. I remember that in 1965 I discussed with Herman Harken in Stuttgart the feasibility of European equivalents to Physical Review Letters and The Physical Review. Pages charges in the journals were almost prohibitively high at the time. I also felt very strongly that physics in Europe should have a clearer voice towards the outside, but also, as a main element of our culture, to the inside of our own continent."

Commercial European publishers were by 1965 well established in physics, and it was wishful to think that much could be done immediately. There were also other issues to tackle, notably the structure of the Society. This was to be defined at further meetings hosted by the IPPS in London (May 1967), by the Institut Battelle in Geneva (January 1968), and by the Czechoslovak Academy of Sciences in Prague (May 1968). About 40 physicists covering most European countries and all fields of physics were to participate in the discussions.

\section{To Federate or Not?}

The Society's first major question of international relationships was clearly within the Society itself. Bernardini had argued in 1966 that EPS should "provide a forum for the discussion of subjects of common interest to all European physicists" and "provide means whereby action can be taken on matters which cannot conveniently be handled by national bodies." Georges Béné, who had been Secretary-General of the Groupement Ampère for 10 years, recalls on page 122 why he proposed an independent, non-federative structure. But several other views were expressed, and papers presented at the London meeting by $\mathrm{Ch}$. Peyrou and by the French Physical Society (which was not represented at the committee's first meeting owing to misdirected invitations) came out strongly in favour of a confederative structure. A working group for a provisional constitution chaired by A. Abragam, the President of the French society, formulated the so-called Abragam compromise to establish a modus vivendi with national physical societies. It stressed that EPS "should not have any negative influence on the national societies"; co-operation was emphasized and the present scheme of direct and indirect membership recommended. This allowed the drawing up of the Constitution and the By-Laws to go ahead.

Recommendations by a second working group under Bernardini were put into effect with the formation of a Bureau comprising Béné, Jansen, Josef-Maria Jauch (who passed away in 1974), Cohen, Wolfgang Gentner, and Bernardini, with Lorette Etienne from Battelle's Advanced Studies Group as the Scientific Secretary working part-time. The Bureau's secretariat was installed in the University of Geneva's Theoretical Physics Department in September 1967, with an assistant supported by the Groupement Ampère (the IPPS had also been eager to host the Bureau so it was decided in May to have a branch office under Cohen in London). The official inauguration took place a year later in the University's Aula Magna following a plenary meeting in the CERN Council Chamber. Bernardini, the first President, described EPS as "a further demonstration of the determination of scientists to work together and make their positive contribution to the strength of European cultural unity." Membership extended to 18 national societies.

So the EPS was born. Etienne was appointed the Executive Secretary at the inauguration and the secretariat soon transferred to Battelle, where it remained until moving in June 1973 into premises in Petit-Lancy vacated by the Institute. The Inaugural Conference The Growth Points of Physics - the first major scientific conference organized by EPS - took place in Florence in April 1969. There were 65 speakers and session chairs, and IUPAP formally endorsed the event. An election of delegates of the Individual Ordinary Members at a General Assembly during the conference completed the first Council, which then held its first session.

\section{Steady Progress}

Bernardini redefined the Society's goals in 1968 as motivations had been moulded to reality during the Society's birth. It is perhaps useful to list these goals and highlight the progress that has been achieved since this should provide strong encouragement to continue. Other contributors will, in some cases, flesh out the details.

- Coordinate meetings and conferences: the EPS now does rather well as the Divisions in fact organize most of the major European events in physics.

- Act as an international forum: this works to some extent, but much more could be done. The EPS should become the natural framework through which large international constituencies for scientific facilities manifest themselves.

- Coordinate European publications: Bernardini thought that the EPS could help the emergence of strong European journals in the various fields of physics, and also provide editorial boards and referees. He felt review journals should be encouraged as an antidote to extreme specialization. A little has been done, 
but we are still far from this ambitious, but most natural, goal. Europhysics Letters, a joint venture between 16 national societies and EPS has shown what can be achieved.

- Limit the proliferation of summer schools: the EPS has not done much in this area.

- Publish a regular bulletin: Europhysics News is succesful and is considered to be a useful journal.

- Foster the teaching of physics: much remains to be done. An Advisory Committee on Physics Education was formed in 1974. It helped publish a newsletter from 1975 to 1984, steer the creation of the European Journal of Physics, conduct surveys of physics teaching in secondary schools (1980) and of tertiary physics education (1984), and promote the use of computers in teaching. The committee became an Action Committee (along with all the other Advisory Committees) in 1989 and was reconstituted in 1993 as an Interdivisional Group to stimulate a broader range of activities. One of the Group's aims is bring an international perspective to physics teaching so the recent creation of the international Amaldi Prize for physics textbooks, which will be ajudicated by the Group, is a significant step. - Encourage the exchange of students and young physicists: Bernardini proposed implementing enabling measures so that young people would have "the same degree of opportunity, the same facilities, independently of the country in which they completed their first period of university studies". An EPS Scholarship scheme to allow advanced graduate students and postdocs to train outside their own countries was proposed in 1977 by B. Giovannini. The first offerings were announced in Europhysics News in December 1978 but in spite of vigorous efforts, the scheme never really took off. The Society soon discovered that large resources were needed for administration as scholarships are complicated, essentially bilateral affairs. Student mobility was taken up again in 1991 with the initiative by Ernst Heer from Geneva to form the European Mobility Scheme for Physics Students that will see its first transfers this year.

\section{... and the New Challenges}

Physics is universal, transcending national specificities and pride; physicists are well aware of the great and numerous advantages which can be found in a lively international collaboration, and they can, perhaps more easily than other scientists, overcome its inherent difficulties. It was also a great challenge to try to ignore, as much as possible, the imposing barrier which had been set across our continent. Creating EPS with member societies and individual members from eastern and western Europe showed a clear willingness to use the bonds provided by physics to establish links and foster collaboration despite great difficulties, which spoke for themselves. Now that the barrier is at long last gone, EPS can capitalise on all the fruitful links which it set up, and on the spirit of collaboration which it helped keep alive. The new expectations are great. Fortunately, there is now a definite willingness to develop cooperation as much as possible. Yet while political difficulties largely belong to the past, many other difficulties have to be overcome. This is the new great challenge.

One of the Society's most important partners is clearly The American Physical Society, with

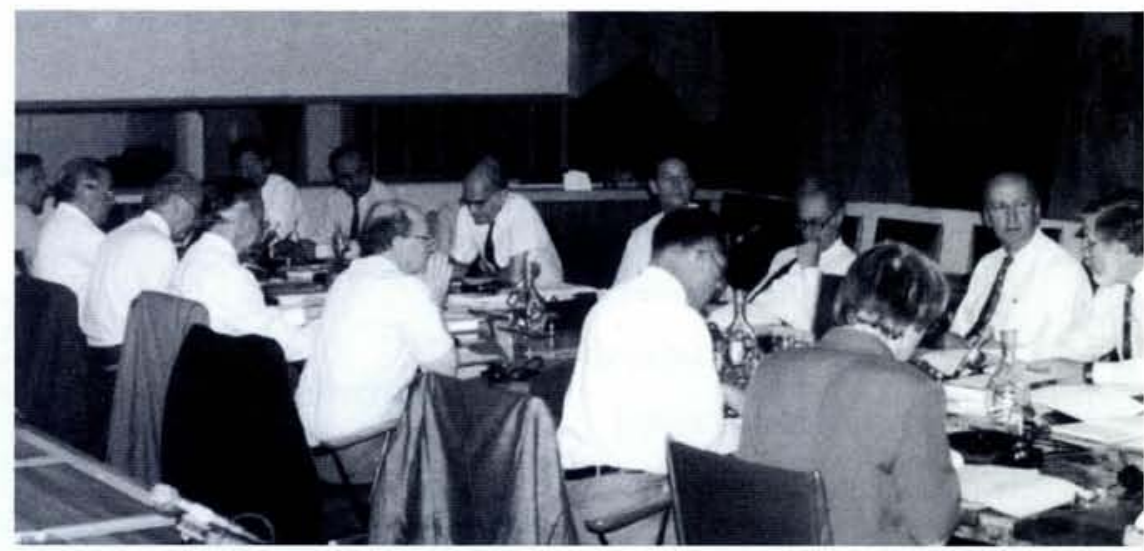

which relations in the past have not been very clear. There may have been a feeling of inferiority as the APS draws important revenues from publications, and APS Division conferences are seen as fascinating successes. Moreover, incorporating eastern Europe was a difficult matter, which stronger ties with the APS could have made more problematic. More generally, the Society's federative element made EPS seem weak in front of a unique APS of continental scale.

But things have changed. Many physicists of my generation, who have lived the scientific recovery of Europe, show much gratitude towards America, where one could learn modern, living physics in the 1950's and 1960's. Not having strong links between EPS and APS appeared odd.

Recent efforts to strengthen relations have met with much goodwill. What could be achieved so far is summarised in presidential letters published in Europhysics News and APS News. Coordination and joint activities were heralded by a "summit" meeting in Budapest in 1992 (covering eastern Europe) and an UNESCO Consultation Meeting in Paris in June 1993 (covering a variety of topics).

Japan has become a scientific and technological giant and the young dragons of Asia are making important contributions. It is natural, therefore, that information should be exchanged with the two physical societies in Japan, and with the Association of Asia-Pacific Societies, with the view to developing joint activities.

EPS of course extends across Europe, and national societies, if they existed, from neighbouring countries were invited to join right from the beginning. Given the scope, there are many national susceptibilities to overcome among people who believe they share a common culture; it is wonderful to see how things work. But there is still much to be done. The Society has developed good relations with major European research organizations. It should increasingly act as their natural partner, advising them on the opinions and aspirations of the physics community at large, especially in the development of large facilities operating at an international level.

A closer association with the European Community through the Commission of the EC in areas such as referee panels, study conferences, student mobilty, and support for literature does not imply negelect of colleagues elsewhere. The Community is becoming an increasingly attractive pole for the whole of Europe, and several non-EC countries already see it as an important element in their futures.
The UNESCO Consultative Meeting with the EPS and the APS at the UNESCO headquarters in Paris on 24-24 June 1993. Representatives of The Physical Society of Japan, the Commission of the European Communities, the International Council of Scientific Unions, the Organization for Economic Cooperation and Development, and other international bodies participated.

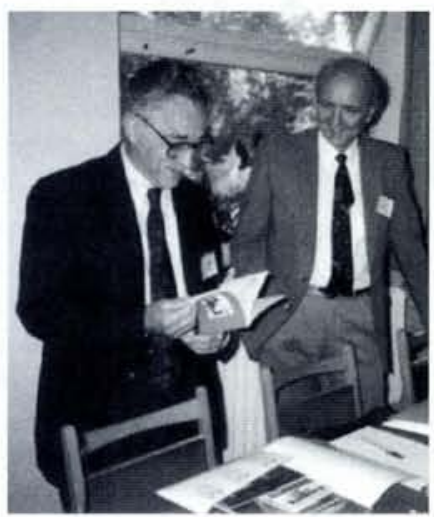

On the left, Ernest Henley, the President of The American Physical Society, and Norbert Kroó, the current EPS President, at the joint meeting of the two societies in Budapest on 25-27 May 1992.

Eddy Lingeman, on the left, Chairman of the Action Committee for Physics and Society, and André Landesman, Chairman of the EastWest Coordination Committee. during a visit to the Commission of the European Communities' Directorate for science (DG-XII) in Brussels in June 1992.

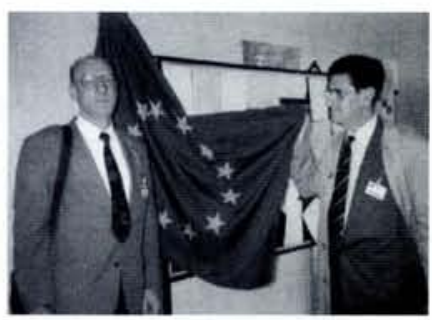

